\title{
Image formation in high-contrast optical systems: the role of polarization
}

\author{
James B. Breckinridge
}

James B. Breckinridge, "Image formation in high-contrast optical systems: the role of polarization," Proc. SPIE 5487, Optical, Infrared, and Millimeter Space Telescopes, (12 October 2004); doi: 10.1117/12.548932

SPIE Event: SPIE Astronomical Telescopes + Instrumentation, 2004, Glasgow, United Kingdom 


\title{
Image Formation in High-Contrast Optical Systems: The role of polarization
}

\author{
James B. Breckinridge \\ Theme Technologist \\ NASA Origins Program \\ Jet Propulsion Laboratory* \\ Pasadena, CA. 91109
}

\begin{abstract}
To find evidence of life in the Universe outside our solar system is one of the most compelling and visionary adventures of the $21^{\text {st }}$ century. The technologies to create the telescopes and instruments that will enable this discovery are now within the grasp of mankind. Direct imaging of a very faint planet around a neighboring bright star requires high contrast or a hypercontrast optical imaging system capable of controlling unwanted radiation within the system to one part in ten to the $11^{\text {th }}$. This paper identifies several physical phenomena that affect image quality in high contrast imaging systems. Polarization induced at curved metallic surfaces and by anisotropy in the deposition process (Smith-Purcell effect) along with beam shifts introduced by the Goos-Hachen effect are discussed. A typical configuration is analyzed, and technical risk mitigation concepts are discussed.
\end{abstract}

\section{INTRODUCTION}

The two remaining unexplored regions of the observable Universe are extremely high spatial resolution and hyper-contrast optics. Telescope systems with extremely high resolution will enable direct imaging across the surface of stars other than our own sun. These high spatial resolution instruments, when combined with hyper contrast optics will enable direct imaging across the surface of planets in other solar systems in our neighborhood. Telescopes with effective apertures of $>1,000$ meters or greater are being studied. A telescope with hyper contrast optics will enable imaging extremely faint sources in the presence of very bright source.

The NASA Astronomical Search for Origins Program, sometimes abbreviated "Origins" consists of 4 missions, each one significantly more complex than the previous and each one requiring incredible new technology - inventions and processes that stretch the imagination. These are the James Webb Space Telescope, the Space Interferometer Mission, Terrestrial Planet Finder, and Life Finder. Origins missions use new windows of high spatial resolution and hyper contrast optical systems to image astronomical objects at micro-arc second resolution and with extremely low backgrounds. One of the mission objectives of the origins program is to detect and characterize exo-solar system planets. Control within the optical system of unwanted radiation at the level of one part in 10 to the $12^{\text {th }}$ will be required ${ }^{1}$.

The unique environment of space without the presence of atmospheric turbulence, molecular and particle scattering will enable unprecedented dynamic range imaging. This same property also enables interferometers in space to yield high-spatial resolution imaging. This paper concentrates on high contrast optical systems.

Today, special purpose high ground-based solar astronomers to study the solar corona regularly use high contrast optics. Astronomical research of the solar corona from the surface of the earth requires control of scattered light to better than 7 orders of magnitude. Forward scattering in the atmosphere is the primary source of the noise photons or scattered light for ground-based astronomy. For observations in space the 
corresponding theoretical limit is forward scattering introduced by dust particles in the solar system ecliptic or the plane in which the planets move around the sun. Measurements indicate that this level of forward scattering is far below that to be expected to be required for the direct imaging of a terrestrial planet.

This paper reports the design principles for a high-contrast optical system, explains how astronomers build special purpose optical systems to image the very faint solar corona in the presence of the very bright solar surface, discusses the effects of polarized light on the image formation process, identifies sources of polarization in high contrast optical systems, provides a methodology for testing optical systems for the effects of polarization, and provides an outline for suggested future work.

It is well known in the optical science and engineering communities ${ }^{2}$ that imaging systems operate as a partial polarizer on the incoming radiation, altering the nature of the wavefront at the image plane and also providing unwanted radiation at the focal plane.

\section{Coronagraph}

\section{HIGH CONTRAST OPTICAL SYSTEMS}

Since this special-purpose telescope system was originally designed in response to a need to image the solar corona, this high contrast imaging optical system has been called a coronagraph. Figure 1 shows a schematic view of a coronagraph optical system. Light from a distant star enters onto an aperture at plane 1 of optical power $\Phi_{1}$ from the left in the drawing. An image of object space is formed at a field stop located at plane 2. The telescope is centered with the star on axis. An amplitude and phase mask is located at this image plane to obscure the bright on-axis star. The dimensions of this spot, that is its size, amplitude, phase transmittance and reflectance profiles are optimized to control the amount of unwanted radiation in the system. To the right of this image plane mask at plane 2 is located a field lens of optical power $\Phi_{3}$. This field lens relays an image of the entrance aperture onto an edge-mask at plane 4. Returning to plane 1: diffraction around the edge of the entrance aperture at plane 1 creates a bright ring of unwanted radiation at the image of the entrance aperture. The ring mask at plane 4 is designed and built to control the amplitude and phase transmittance and reflectivity of the unwanted radiation diffracted around the edge of the entrance aperture at plane 1 . The optic at plane 5 relays the image plane 2 onto the final image plane 6 where the detector array is located. 


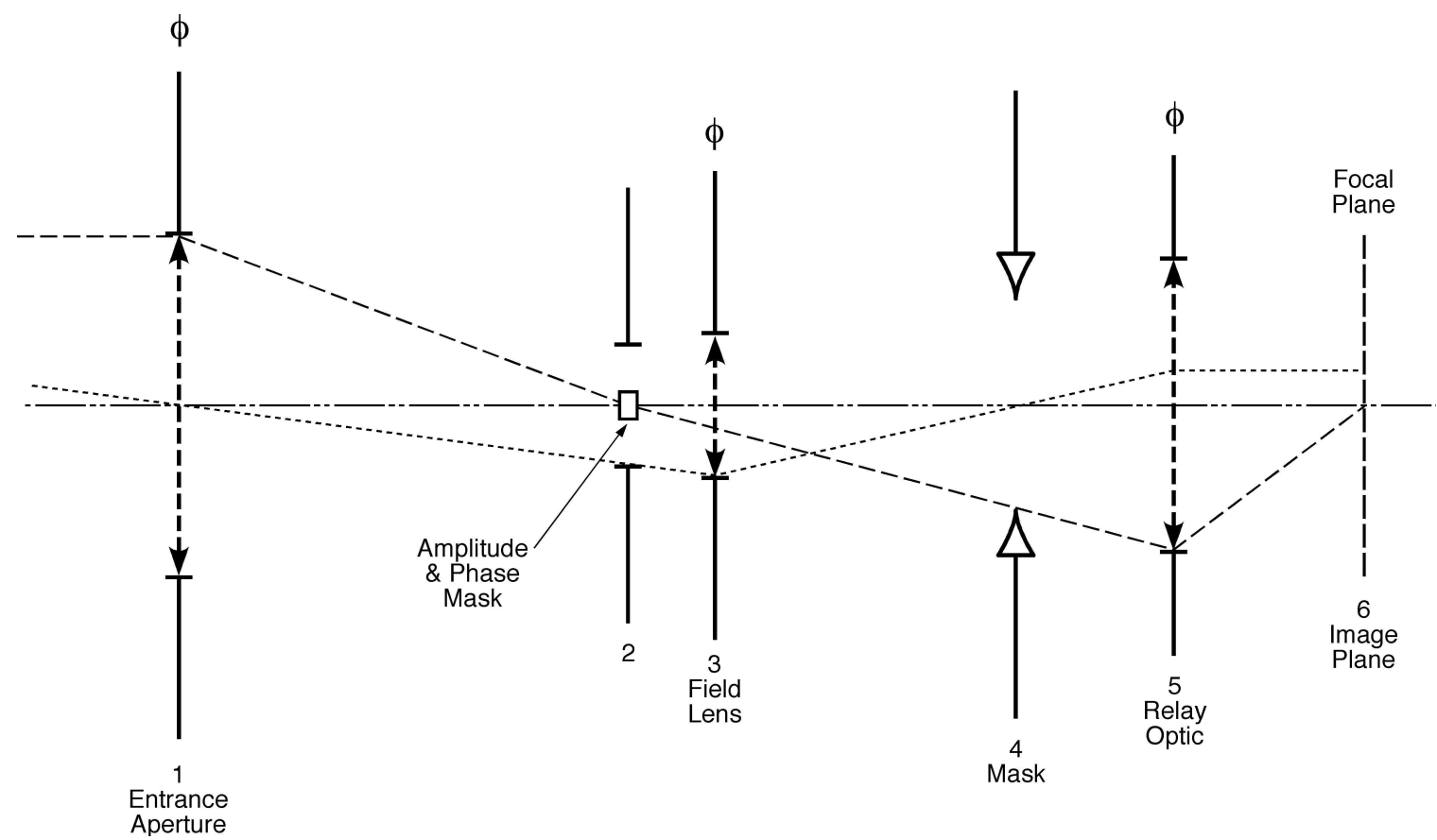

Figure 1. Schematic view of a coronagraph or a high-contrast optical system.

The profile of the mask at plane 2 in terms of the complex amplitude and phase transmittance as a function of position of this surface and the profile of the mask at plane 2 in terms of the complex amplitude and phase transmittance as a function of position across this surface are changed to minimize the unwanted radiation at the image plane 6 , while maintaining the required image quality ${ }^{3}$.

\section{Mirrors and Polarization}

Telescope primary mirror at plane 1 as shown schematically in figure 1 are curved concave to reflect and concentrate the radiation and to form a high quality image of the object space onto an occulting mask and to the far right in the figure, onto the image focal plane array.

Born and $\mathrm{Wolf}^{4}$ showed that if light is incident on a reflecting mirror at an angle, the reflectivity of the light polarized in the plane perpendicular to the plane of incidence is not equal to the reflectivity of the light polarized in the plane parallel to the plane of incidence.

A ray from the object, which reflects from the edge (marginal ray) of the mirror to the focus, has reflected off the mirror at an angle larger than those rays closer to the vertex of the mirror. As the F-number decreases, the marginal ray angle deviation increases, where the marginal ray angle is simply arcsine (1.0/2.F\#), where F\# is the F-number of the imaging system. Consequently there will be a change in the polarization reflectivity across the pupil and the pupil is said to be polarization-dependent apodized.

A perfectly deposited thin film on an optical substrate as a function of the angle of reflection is now considered. We calculate of the reflectivity in both $\mathrm{s}\left(\mathrm{R}_{\perp}\right)$ and $\mathrm{p}\left(\mathrm{R}_{/ /}\right)$polarizations of light incident on ideal aluminum, silver and gold metal films as a function of angle of incidence and of wavelength.

Consider an ideal highly reflecting metal thin film. F. Abeles ${ }^{5},{ }^{6}$ showed that if a ray traveling in a dielectric medium of index $\mathrm{n}_{0}$ is incident onto a conducting (metal) substrate of complex index of refraction: $\mathrm{n}$ $-\mathrm{jk}$ at an angle of incidence of $\phi_{0}$ then the ratio of the reflectivity in parallel light to that in perpendicular, is given by: 


$$
\frac{R_{/ /}}{R_{\perp}}=\frac{\left(p \cos \phi_{0}-n_{0} \sin ^{2} \phi_{0}\right)^{2}+q^{2} \cos ^{2} \phi_{0}}{\left(p \cos \phi_{0}+n_{0} \sin ^{2} \phi_{0}\right)^{2}+q^{2} \cos ^{2} \phi_{0}},
$$

Where the substitution:

$$
(p-j q)^{2}=(n-j k)^{2}-n_{0}^{2} \sin ^{2} \phi_{0}
$$

has been made. The individual (complex) values for $\mathrm{p}$ and $\mathrm{q}$ in terms of $\mathrm{n}$ and $\mathrm{k}$ is found by setting the real part of the left hand side of equation 2 to the real part of the right hand side of equation 1, and by setting the imaginary part of the LHS of equation 2 to the imaginary part of the RHS of equation 2. For the uninteresting case, at normal incidence, $\phi_{0}=0.0$, then $\mathrm{p}=\mathrm{n}$ and $\mathrm{q}=\mathrm{k}$. Solving equation 2 gives the system of equations:

$$
p^{2}-q^{2}=n^{2}-k^{2}-n_{0}^{2} \sin ^{2} \phi_{0}
$$

and

$p q=n k$.

We will now apply equations 6 and 8 from Breckinridge and Oppenheimer ${ }^{7}$ and use the wavelength dependent optical constants from Palik ${ }^{8}$, to calculate the reflectivity for the transverse electromagnetic (te) wave, $R_{\perp}$, and the reflectivity for the tm wave, $\left.R_{/ /}\right)$. The polarization percentage (\%) is given by: $\left(R_{\perp}\right)$ and $\left(\mathrm{R}_{/ /}\right)$

$$
\% \text { Polarized }=\left(\mathrm{R}_{\perp}-\mathrm{R}_{/ /}\right) /\left(\mathrm{R}_{\perp}+\mathrm{R}_{/ /}\right)
$$

Equation 3.

\begin{tabular}{|c|c|c|c|c|c|c|c|}
\hline & $\begin{array}{l}\frac{\circ}{\circ} \\
\text { Polarized }\end{array}$ & $\mid \begin{array}{l}\frac{\circ}{0} \\
\text { Polarized }\end{array}$ & $\mid \begin{array}{l}\% \\
\text { Polarized }\end{array}$ & $\mid \begin{array}{l}\% \\
\text { Polarized }\end{array}$ & $\mid \begin{array}{l}\frac{\circ}{0} \\
\text { Polarized }\end{array}$ & $\begin{array}{l}\circ \\
\text { Polarized }\end{array}$ & 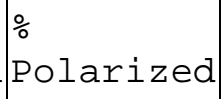 \\
\hline $\begin{array}{l}\text { Angle of } \\
\text { Incidence } \\
\text { in } \\
\text { Degrees }\end{array}$ & $300 \mathrm{~nm}$ & $400 \mathrm{~nm}$ & $500 \mathrm{~nm}$ & $600 \mathrm{~nm}$ & $700 \mathrm{~nm}$ & $800 \mathrm{~nm}$ & $1000 \mathrm{~nm}$ \\
\hline 0.00 & 0.00 & 0.00 & 0.00 & 0.00 & 0.00 & 0.00 & 0.00 \\
\hline 4.00 & 0.33 & 0.27 & 0.20 & 0.02 & 0.01 & 0.01 & 0.00 \\
\hline 8.00 & 1.32 & 1.09 & 0.80 & 0.09 & 0.03 & 0.03 & 0.02 \\
\hline 10.00 & 2.06 & 1.70 & 1.25 & 0.14 & 0.05 & 0.04 & 0.03 \\
\hline 16.00 & 5.27 & 4.37 & 3.17 & 0.37 & 0.14 & 0.11 & 0.08 \\
\hline 22.00 & 9.97 & 8.26 & 5.94 & 0.70 & 0.26 & 0.21 & 0.15 \\
\hline 30.00 & 18.45 & 15.31 & 10.84 & 1.30 & 0.50 & 0.39 & 0.28 \\
\hline
\end{tabular}

Table 1 below shows this ratio. as a function of wavelength for a gold highly reflecting metal coatings on mirrors for a series of marginal ray angles 0 and 30 degrees, from 300 to $1000 \mathrm{~nm}$. Calculated using equation 3. 
Table 2 below shows this ratio as a function of wavelength for an aluminum highly reflecting metal coatings on mirrors for a series of marginal ray angles 0 and 30 degrees, from 300 to $1,000 \mathrm{~nm}$. Calculated using equation 3 .

\begin{tabular}{|c|c|c|c|c|c|c|c|}
\hline & $\begin{array}{l}\circ \\
\text { Polarized }\end{array}$ & $\mid \frac{\circ}{0}$ Polarized & $\left|\begin{array}{l}\frac{\circ}{0} \\
\text { Polarized }\end{array}\right|$ & $\mid \frac{\circ}{0}$ Polarized & $\mid \begin{array}{l}\frac{\circ}{0} \\
\text { Polarized }\end{array}$ & $\mid \begin{array}{l}\frac{\circ}{0} \\
\text { Polarized }\end{array}$ & $\mid \frac{\circ}{0}$ Polarized \\
\hline $\begin{array}{l}\text { Angle of } \\
\text { Incidence } \\
\text { in } \\
\text { Degrees }\end{array}$ & $300 \mathrm{~nm}$ & $400 \mathrm{~nm}$ & $500 \mathrm{~nm}$ & $600 \mathrm{~nm}$ & $700 \mathrm{~nm}$ & $800 \mathrm{~nm}$ & $1000 \mathrm{~nm}$ \\
\hline 0.00 & 0.00 & 0.00 & 0.00 & 0.00 & 0.00 & 0.00 & 0.00 \\
\hline 4.00 & 0.02 & 0.02 & 0.02 & 0.02 & 0,03 & 0.04 & 0.02 \\
\hline 8.00 & 0.09 & 0.08 & 0.08 & 0.09 & 0.12 & 0.14 & 0.08 \\
\hline 10.00 & 0.14 & 0.12 & 0.13 & 0.14 & 0.18 & 0.23 & 0.13 \\
\hline 16.00 & 0.35 & 0.31 & 0.33 & 0.37 & 0.48 & 0.59 & 0.34 \\
\hline 22.00 & 0.66 & 0.60 & 0.64 & 0.71 & 0.91 & 1.12 & 0.65 \\
\hline 30.00 & 1.24 & 1.13 & 1.20 & 1.35 & 1.73 & 2.12 & 1.24 \\
\hline
\end{tabular}


Table 3 below shows this ratio as a function of wavelength for a silver highly reflecting metal coatings on mirrors for a series of marginal ray angles 0 and 30 degrees, from 300 to $1,000 \mathrm{~nm}$. Calculated using equation 3.

\begin{tabular}{|c|c|c|c|c|c|c|c|}
\hline & $\begin{array}{l}\circ \\
\text { Polarized }\end{array}$ & $\mid \begin{array}{l}\frac{\circ}{0} \\
\text { Polarized }\end{array}$ & $\mid \begin{array}{l}\% \\
\text { Polarized }\end{array}$ & $\mid \begin{array}{l}\circ \\
\text { Polarized }\end{array}$ & $\mid \begin{array}{l}\% \\
\text { Polarized }\end{array}$ & $\mid \begin{array}{l}\frac{\circ}{0} \\
\text { Polarized }\end{array}$ & $\mid \begin{array}{l}\circ \\
\text { Polarized }\end{array}$ \\
\hline $\begin{array}{l}\text { Angle of } \\
\text { Incidence } \\
\text { in } \\
\text { Degrees }\end{array}$ & $300 \mathrm{~nm}$ & $400 \mathrm{~nm}$ & $500 \mathrm{~nm}$ & $600 \mathrm{~nm}$ & $700 \mathrm{~nm}$ & $800 \mathrm{~nm}$ & $1000 \mathrm{~nm}$ \\
\hline 0.00 & 0.00 & 0.00 & 0.00 & 0.00 & 0.00 & 0.00 & 0.00 \\
\hline 4.00 & 0.44 & 0.02 & 0.01 & 0.00 & 0.00 & 0.00 & 0.00 \\
\hline 8.00 & 1.76 & 0.08 & 0.02 & 0.02 & 0.01 & 0.01 & 0.01 \\
\hline 10.00 & 2.76 & 0.12 & 0.04 & 0.03 & 0.02 & 0.02 & 0.02 \\
\hline 16.00 & 7.10 & 0.31 & 0.09 & 0.07 & 0.06 & 0.05 & 0.04 \\
\hline 22.00 & 13.53 & 0.57 & 0.18 & 0.13 & 0.11 & 0.09 & 0.08 \\
\hline 30.00 & 25.36 & 1.03 & 0.33 & 0.24 & 0.20 & 0.17 & 0.16 \\
\hline
\end{tabular}

Combining the angles of incidence over the entire telescope beam with the polarization calculations for a given coating results in an apodization function being imposed on the telescope beam. This apodization function is greatest at the beam edge (the marginal ray). The apodization tapers to zero at the precise center of the beam, which is not obscured by the secondary mirror support structure in an optimal off-axis design ${ }^{9}$. The apodization function is also a function of wavelength. The white-light point spread function of Strehl ratio will be larger than that predicted by diffraction theory because of the polarization-apodization introduced by the white-light image formation process using powered (curved) optical elements. It is important to note that in order to reduce the effect of this apodization, telescopes must be designed with long focal lengths (large Fnumbers).

\section{Imperfect Metal Thin Films}

It has been recognized for years that the scattered light performance of the large primary mirror strongly depends on the smoothness of the mirror substrate and much effort has gone into understanding the engineering processes to create a super-smooth surface. The large area-reflecting surface of these substrates, however, does not have the quality specular reflectance required for the mirror. Thus, the substrate must be coated with the highly reflecting metal thin film. If this thin metal film is perfectly isotropic then the limiting effects discussed in section 2 will dominate. Unfortunately, metal thin films over the large areas required for astronomy are not, in general isotropic (amorphous).

A qualitative understanding of the effects of metal thin film inhomogenieties on system scattered-light is provided by analysis of the Smith-Purcell Effect ${ }^{10},{ }^{11}$.

An electromagnetic light wave incident on a metal surface is known to induce an oscillating electronic charge inside the highly conducting metal film on the mirror substrate. The period of oscillation is characteristic of the frequency of the source; that is about $10 \mathrm{E} 14 \mathrm{~Hz}$. If this highly conducting metal has 
isotropic conductance over its entire volume, then the images formed by the mirror will be, "perfect" within the limits of diffraction produced by the limits of the clear aperture.

In one of the earlier studies ${ }^{12}$ of this phenomenon showed that metal thin films are not isotropic. He determined that the degree of polarization introduced by a reflecting Aluminum metal surface depended on the angle of deposition of the metal onto that surface. Horowitz also showed that the microscopic grain structure of the deposited Aluminum metal was not isotropic and varied across the surface: being columnar in some regions and amorphous in others. This anisotropy causes the electronic conductance to vary across the surface and may cause wavefront deformations that dominate the system performance.

A review and analysis ${ }^{13}$ of the micro structural induced anisotropy in optical thin films demonstrated their presence. Reflection anisotropy ${ }^{14}$ in telescope mirror coatings has been examined. Measurements ${ }^{15}$ of the optical anisotropy in thin films deposited obliquely were made in situ observations and computer models were developed to explain his observations.

Ironically, very little work has gone into optimal deposition of metal thin films onto these supersmooth surfaces (except for small optics used in X-ray imaging devices) ${ }^{16}$. The imperfections in the metal thin films generally manifest themselves in the form of unwanted, usually columnar, structure in the film after it has been deposited on the optical substrate surface: These films are not uniformly amorphous metal, as has been assumed by many opticians and telescope manufacturers. High spatial frequency imperfections in metal thin films result in scattering light through large angles in the optical system. If a large area, say $20 \%$ of its surface area contains an anisotropic structure, through preferential polarization, or preferential conduction in certain directions in the metal coating, then unwanted radiation may be scattered into the field of view of a planet.

\section{How important is isotropy?}

To the author's best knowledge, the effects of coating anisotropies on polarization reflectivity apodization has never been measured in image forming systems. The procedure described here will provide important information on the magnitude of the effect.

\section{Measurement set-up and procedures}

A testbed is examined here that will provide the data needed to understand the role and mitigation of coating anisotropies in high contrast optical systems. 
Figure 2 below shows a diagram of the basic optical test set up to measure the polarization reflectivity across areas of the mirror.

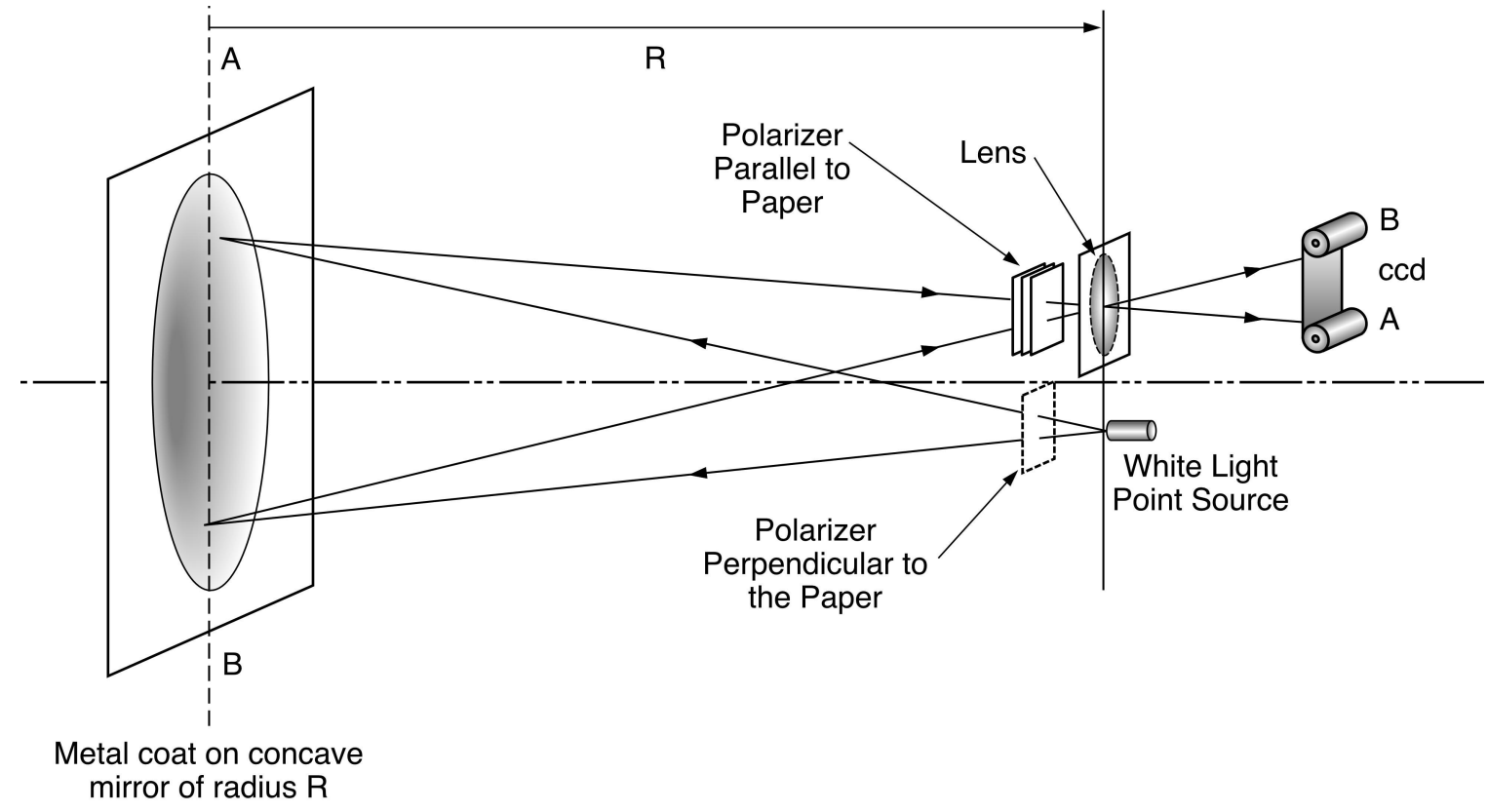

A spherical mirror $(\mathrm{AB})$ coated with a highly reflective metal is located with its center of curvature (at distance $\mathrm{R}$ from the vertex) to the right in the drawing above. Just below the center of curvature we locate the end of a white-light multimode fiber that forms a point source for a white light wavefront to fill the aperture of the mirror AB. Alternatively a 100-watt "point-a-lite" or vacuum arc light can be used to generate a point source of unpolarized nature. Note that for both the vacuum arc and the fiber bundle, stress birefringence may introduce some circular polarization, but the effect will be small and it will be neglected. The power supply for the white-light source will need to be regulated so the lamp has near-constant radiant exitence over periods of a few 10's of minutes. The source of radiation should be as close to the center of curvature point as possible so that each ray associated with the spherically expanding wavefront strikes the surface $\mathrm{AB}$ at normal incidence. All rays from the center of curvature strike the large primary mirror at normal incidence. This arrangement ensures that reflectivity anomalies observed across the surface AB will not be the result of the F\# of the system, but rather caused by surface-reflectivity processing effects.

The wavefront expands from the white-light source, fills the mirror surface and reflects back to converge to a point within the lens of a CCD camera located just above the center of curvature. This CCD camera lens re-images the mirror surface $\mathrm{AB}$ (located in the near-field) on to a $\mathrm{CCD}$ chip. A sheet polarizer is placed in a stand where it can be rotated about an axis normal to the surface with some precision (0.5-degree). The stand that holds the sheet polarizer will need to be placed in a fixture so that tilt of the surface can be adjusted. Two assemblies of this type are required, one in front of the source that generates the expanding white-light wavefront and the other located in front of and covering the lens of the CCD camera.

The commercial sheet polarizer will have good polarization extinction (1:400) over a relatively broadband of $\sim 1,000$ angstroms in the "visible", depending on which film is ordered. An optical filter (wedged and tilted) that passes only radiation across this full band will be needed to be placed over the lens of the CCD camera. The focal length of the CCD lens and the front focal distance and back focal distance should be adjusted so a sharp image of the mirror AB fills the CCD field of view. 


\section{Summary}

This paper demonstrates that polarization is an important issue in space-based high contrast imaging systems. Since orthogonally polarized light does not interfere to contribute to the image formation process. Therefore the telescope system point spread function size is not given by the classical diffraction "Strehl" ratio, but rather is about $10 \%$ larger for $\mathrm{F} \#=1$ systems.

\section{Acknowledgments}

The author acknowledges helpful discussion with Ben Oppenheimer of the American Museum of Natural History, and Stuart Shaklan of the Jet Propulsion Laboratory.

This research was carried out at the Jet Propulsion Laboratory, California Institute of Technology, under a contract with the National Aeronautics and Space Administration.

\section{REFERENCES}

${ }^{1}$ Woolf, N. and Angel, R. 1998, Ann. Rev. Astron. Astrophys., 36, 507-510

${ }^{2}$ Breckinridge, J. and B. Oppenheimer, 2004 ApJ 600: 1091-1098

${ }^{3}$ Breckinridge, J. Kuper, T., \& Shack, R. 1984 Opt. Eng., 23, 816-821.

${ }^{4}$ Born, M. and Wolf, E., 1980, Principals of Optics, $6^{\text {th }}$ ed. (New York: Wiley)

${ }^{5}$ Abeles, F., 1957, J. Opt. Soc. Amer., 47, 473

${ }^{6}$ Abeles, F., 1963, Progress in Optics, Volume II, E. Wolf, ed., (Dordrecht: Elsevier Pub.)

${ }^{7}$ Breckinridge, J. and B. Oppenheimer, 2004 ApJ 600: 1091-1098

${ }^{8}$ Palik, E., 1985, Handbook of Optical Constants of Solids, (New York: Academic Press)

${ }^{9}$ Tokunaga, A., Ftaclas, C., Kuhn, J. R., Baudoz, P., 2002, in Brown Dwarfs, IAU Conf. Proc.,

${ }^{10}$ Smith, S. J. and Purcell, E. M., 1953, Phys. Rev., 92, 1069

${ }^{11}$ Haeberle, O., Rullhusen, P., Salome, J-M. and Maerne, N., 1997, Phys. Rev. E, 55, 4675

${ }^{12}$ Horowitz, F., 1983, Ph.D. Thesis, University of Arizona, Tucson

${ }^{13}$ Dirks, A. G. and Leamy, H. J., 1977, Thin Solid Films, 47, 219

${ }^{14}$ Gee, J. R. and Wilson, P. W., 1986, J. Vac. Sci. Tech., 4, 1875

${ }^{15}$ Hodgkinson, I., 1991, Appl. Opt., 30, 1303

${ }^{16}$ Windt, D. L. and Waskiewicz, W. K., 1994, J. Vac. Sci. Tech. B, 12, 3826 